\title{
Study on Dynamics of Saponification Process Using State Space Model
}

\author{
Khalid Mokhlif Mousa, Zainab Essaim Dawood \\ Department of Chemical Engineering, Al-Nahrain University, Baghdad, Iraq
}

Email address:

drkhalidalzobai@yahoo.com (K. M. Mousa), zaynab.dawood@yahoo.com (Z. E. Dawood)

To cite this article:

Khalid Mokhlif Mousa, Zainab Essaim Dawood. Study on Dynamics of Saponification Process Using State Space Model. American Journal of Chemical Engineering. Vol. 3, No. 4, 2015, pp. 52-57. doi: 10.11648/j.ajche.20150304.11

\begin{abstract}
Due to multi input multi output variables affected the continuous stirred tank reactor its worthy to use the state space model to study the dynamics of this system. In this work, a saponifecation process was studded on which Ethel acetate was reacted with sodium hydroxide to produced sodium acetate and ethanol. one litter operation volume CSTR was used, the flow rate of Ethel acetate and cooling water were toke as disturbance variables and the concentration of sodium acetate and the temperature of reactor as response variables. The results show improvement compared with linearized model.
\end{abstract}

Keywords: CSTR, Sodium acetate, State Space Model

\section{Introduction}

Continuous stirred tank reactors (CSTR) are the most basic of the continuous reactors used in chemical processes. Continuous stirred-tank reactors (CSTRs) are open systems, where material is free to enter or exit the system, that operate on a steady-state basis, where the conditions in the reactor don't change with time. Reactants are continuously introduced into the reactor, while products are continuously removed. CSTRs are very well mixed, so the contents have relatively uniform properties such as temperature, density, etc. throughout[1]. State-space equations, or simply state equations, is a description in the time domain which may be applied to a very wide category of systems, such as linear and nonlinear systems, time-invariant and time-varying systems, systems with nonzero initial conditions, and others. The term state of a system refers to the past, present, and future of the system. From the mathematical point of view, the state of a system is expressed by its state variables. State equations is a description which relates the following four elements: input, system, state variables, and output. In contrast, the differential equations, the transfer function, and the impulse response relate three elements: input, system, and output-wherein the input is related to the output via the system directly (i.e., without giving information about the state of the system). It is exactly for this reason that these three system descriptions are called input-output descriptions [2].
The theory and practice of control engineering since the mid-1900s provide some perspective for the material. In the 1940s and 1950s, systems were represented in the frequency domain by transfer functions. Analysis techniques involving Evans root locus plots, Bode plots, Nyquist plots, and Nichols charts. The $1960 \mathrm{~s}$ and $1970 \mathrm{~s}$ witnessed a fundamental paradigm shift from the frequency domain to the time domain. Systems were represented in the time domain by a type of differential equation called a state equation. Performance and robustness specifications also were specified in the time domain, often in the form of a quadratic performance index. Key advantages of the state-space approach were that a time-domain formulation exploited the advances in digital computer technology and the analysis and design methods were well-suited to multiple-input, multipleoutput systems. Moreover, feedback control laws were calculated using analytical formulas, often directly optimizing a particular performance index. The 1980's and 1990's were characterized by a merging of frequency domain and time-domain viewpoints. Specifically, frequency-domain performance and robustness specifications once again were favored, coupled with important theoretical breakthroughs that yielded tools for handling multiple-input, multipleoutput systems in the frequency domain. Further advances yielded state-space time-domain techniques for controller synthesis. In the end, the best features of the preceding decades were merged into a powerful, unified framework [3]. L.M. Patnaik et al. developed a state space model of a tubular ammonia reactor which is the heart of an ammonia plant in a 
fertiliser complex. The lumped model is chosen such that the steady state temperature at the exit of the catalyst bed computed from the simplified state space model is close enough to the one computed from the nonlinear steady state model. The model developed is very useful for the design of continuous/discrete versions of single variable/multivariable control algorithms [4]. Neil Shephard suggested the use of simulation techniques to extend the applicability of the usual Gaussian state space filtering and smoothing techniques to a class of non-Gaussian time series models. This allows a fully Bayesian or maximum likelihood analysis of some interesting models, including outlier models, discrete Markov chain components, multiplicative models and stochastic variance models[5]. Jeremy F. Burri et al. presented a methodology for the construction of the attainable region (AR) and for the synthesis of globally optimal reactor networks employing isothermal, steady-state, plug flow reactors (PFR), continuous-stirred tank reactors (CSTR) as well as mixing. The problem is solved using the novel Infinite Dimensional State-space (IDEAS) approach. Point wise identification of the reactor network's attainable region is shown to be equivalent to the solution of an infinite dimensional linear program. A finite dimensional approximation strategy is presented [6]. Tapani Raiko et al. studied the learning of nonlinear state space models for a control task. Variational Bayesian learning provides a framework where uncertainty is explicitly taken into account and system identification can be combined with modelpredictive control. Three different control schemes are used. One of them, optimistic inference control, is a novel method based directly on the probabilistic modelling [7]. C. Or et al. and John Kim studied Finite-dimensional state-space approximations of the Orr-Sommerfeld equation for plane Poiseuille flow with boundary input and output are discretized to state-space models using two spectral techniques. The models are compared for accuracy and discussed in the context of metrics important for a nonnormal dynamical system. Both state-space models capture the sensitivity behavior of the pole-zero perturbations and the pseudo spectrum properties [8]. R.N. Methekar et al. innovated form of state space models that facilitate the development of advanced control algorithms such as linear quadratic Gaussian (LQG) and model predictive control (MPC), and provide improved disturbance rejection necessary for these applications. Demonstrated the applications of such model based algorithms via simulations involving a distributed along-the-channel model of the PEMFC, and also presented experimental validation on a PEMFC setup [9]. Siem J. Koopman et al. reviewed and provided some adequate details and guidance for the adaptation of state space methods in univariate and multivariate time series analysis. Provide more detailed discussions for linear Gaussian model formulations and more concise reviews for nonlinear and non-Gaussian departures [10]. The transfer function approach is sufficient to calculate the response of linear control systems. The state-space approach is especially valuable in the field of optimal control of linear or nonlinear systems. The differential equations can be written in the form

$$
\begin{aligned}
& x \dot{x(t)}=A x(t)+B u(t) \\
& \dot{y(t)}=C y(t)+D u(t)
\end{aligned}
$$

Equation (1) is a system of first-order differential equations and is known as the state equation of the system. The vector $x(t)$ is the state vector, and $u(t)$ is the input vector. Equation (2) is referred to as the output equation. $A$ is called the state matrix, $B$ the input matrix, $C$ the output matrix, and $D$ is the direct transition matrix. One advantage of the state space method is that the form lends itself easily to the digital and analog computation methods of solution. Further, the state space method can be easily extended to the analysis of nonlinear systems. State equations may be obtained from an $n t h$ order differential equation or directly from the system model by identifying appropriate state variables. To illustrate the first method, consider an nth order linear plant model described by the differential equation

$$
\frac{d^{n} y}{d t^{n}}+a_{n-1} \frac{d^{n-1} y}{d t^{n-1}}+\cdots+a \frac{d y}{d t}+a_{o} y=u(t)
$$

Where $y(t)$ is the plant output and $u(t)$ is the plant input. A state model for this system is not unique but depends on the choice of a set of state variables. A useful set of state variables, referred to as phase variables, is defined as:

$$
x_{1}=y ; x_{2}=\dot{y} ; x_{3}=\ddot{y} ; \ldots ; x_{n}=\frac{d^{n-1} y}{d t^{n-1}}
$$

Taking derivatives of the first $n$ - 1 state variables, we have

$$
\dot{x_{1}}=x_{2} ; \dot{x_{2}}=x_{3} ; \ldots ; x_{n-1}^{\cdot}=x_{n}
$$

In addition, $\dot{x_{n}}$ comes from rearranging Eq. (3) and substituting from Eq. (4):

$$
\dot{x_{n}}=-a_{o} x_{1}-a_{1} x_{2}-\cdots-a_{n-1} x_{n}+u(t)
$$

In matrix form, this looks like

$$
\begin{gathered}
{\left[\begin{array}{c}
\dot{x_{1}} \\
\dot{x_{2}} \\
\vdots \\
x_{n-1} \\
\dot{x_{n}}
\end{array}\right]=\left[\begin{array}{ccrrl}
0 & 1 & 0 & \ldots & 0 \\
0 & 0 & 1 & \ldots & 0 \\
\vdots & \vdots & \vdots & \ddots & \vdots \\
0 & 0 & 0 & \ldots & 1 \\
-a_{o} & -a_{1} & -a_{2} & \ldots & -a_{n-1}
\end{array}\right]} \\
{\left[\begin{array}{c}
x_{1} \\
x_{2} \\
\vdots \\
x_{n-1} \\
x_{n}
\end{array}\right]+\left[\begin{array}{c}
0 \\
0 \\
\vdots \\
0 \\
1
\end{array}\right] u(t)}
\end{gathered}
$$

Thus the output equation is simply

$$
y=\left[\begin{array}{lllll}
1 & 0 & 0 & \ldots & 0
\end{array}\right] x
$$

A transfer function that relates an output variable to an input variable represents an $n^{\text {th }}$-order differential equation. In the state-space representation, the $\mathrm{n}^{\text {th }}$-order differential equation is written as $\mathrm{n}$ first-order differential equations in 
terms of $\mathrm{n}$ state variables [11]. The matrix differential equation

$$
\dot{\mathrm{x}}=\mathrm{Ax}+\mathrm{Bu}
$$

used to describe a control system by the state-space method can be solved for the vector of state variables $(\mathrm{x})$ by use of the transfer function matrix. It consists of a matrix of transfer functions that relate the state variables to the inputs. The transfer function matrix serves the same purpose in a multiple-input multiple-output system as the transfer function does for a single-input single-output system. The transfer function matrix is obtained from the matrix differential equatien by application of Laplace transforms[12]. The aim of this work is to study the dynamics of saponification process using state space model. A detail of the reaction and experimental works is shown in [13].

\section{Results and Discussion}

The dynamic behavior of saponification process in continuous stirred tank reactor was studied experimentally using step change in the manipulated variables (Ethel acetate flow rate and water coil flow rate $\left(\mathrm{F}\right.$ and $\left.\mathrm{F}_{\mathrm{ace}}\right)$ ) and examine the transient response of sodium acetate and reaction temperature as control variables. The matrics described the dynamics of saponification process as fallow:

$$
C_{D}=\left[\begin{array}{cc}
\frac{0.022 e^{-0.33 s}}{15 S+1} & \frac{5 e^{-0.33 s}}{21 S+1} \\
\frac{0.0056 e^{-6 s}}{21 S+1} & \frac{5.9 e^{-0.33 s}}{21 S+1}
\end{array}\right] F
$$

The reactions inside the reactor can be described by following reaction :-

Ethyl acetate $(\mathrm{A})+$ Sodium hydroxide $(\mathrm{B}) \rightarrow$ Sodium acetate (D) + Ethanol (E)

A detailed of the system was shown in [13].

The state space was found by MATLAB and converted to Block diagram to compare with the open loop MIMO system. The above matrics can be represented in MATLAB as fallow:

$$
\begin{aligned}
& >>\text { den }=\left\{\left[\begin{array}{ll}
15 & 1
\end{array}\right],\left[\begin{array}{ll}
21 & 1
\end{array}\right] ;\left[\begin{array}{ll}
21 & 1
\end{array}\right],\left[\begin{array}{ll}
21 & 1
\end{array}\right]\right\} \\
& >>\text { num }=\{0.022,5 ; 0.0056,5.9\} \text {; } \\
& >\mathrm{p}=\mathrm{tf} \text { (num,den) } \\
& \mathrm{p}= \\
& \text { From input } 1 \text { to output. } \\
& 1: \frac{0.022}{15 s+1} \\
& \text { 2: } \frac{0.0056}{21 s+1}
\end{aligned}
$$

From input 2 to output.

$$
\begin{aligned}
& \text { 1: } \frac{5}{-----} \\
& 2: \quad \frac{5.9}{21 s+1}
\end{aligned}
$$

Continuous-time transfer function. $>$ p.iodelay $=[0.330 .33 ; 60.33]$; $\mathrm{p}=$ From input 1 to output.

$$
\begin{aligned}
& \text { 1: } \exp \left(-0.33^{*} s\right) * \begin{array}{c}
0.022 \\
-15 s+1
\end{array}
\end{aligned}
$$

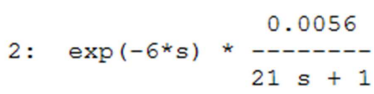

From input 2 to output.

$$
\begin{array}{rl}
1: \exp (-0.33 * s) * \frac{5}{------} & \\
21 s+1 & 5.9 \\
2: \exp (-0.33 * s) * \frac{----}{-} & 21 s+1
\end{array}
$$

Continuous-time transfer function.

$$
>>\text { Sys }=\text { ss (p) }
$$

sys

$$
\mathrm{a}=
$$

$\begin{array}{rlll} & \mathrm{x} 1 & \mathrm{x} 2 & \mathrm{x} 3 \\ \mathrm{x} 1 & -0.06667 & 0 & 0 \\ \mathrm{x} 2 & 0 & -0.04762 & 0 \\ \mathrm{x} 3 & 0 & 0 & -0.0 \\ \mathrm{~b}= & & & \end{array}$

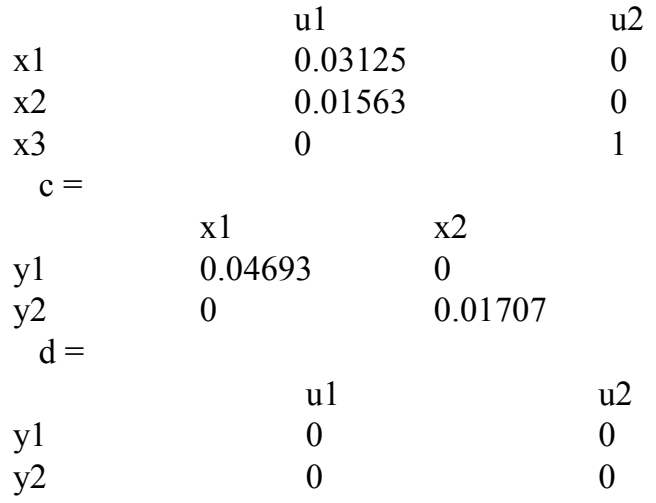

From Continuous-Time State-Space Model of MATLAB answer is the state space matrics

$$
\begin{gathered}
\begin{array}{c}
\dot{X}_{1} \\
\dot{X}_{2} \\
\dot{X_{3}}
\end{array}=\left[\begin{array}{ccc}
-0.06667 & 0 & 0 \\
0 & -0.04762 & 0 \\
0 & 0 & -0.04762
\end{array}\right] \begin{array}{l}
X_{1} \\
X_{2} \\
X_{3}
\end{array} \\
+\left[\begin{array}{cc}
0.03125 & 0 \\
0.9563 & 0 \\
0 & 1
\end{array}\right] U_{1} \\
U_{2} \\
Y_{1}=\left[\begin{array}{ccc}
0.04693 & 0 & 0.2381 \\
0 & 0.01707 & 0.281
\end{array}\right] \begin{array}{l}
X_{1} \\
X_{2} \\
Y_{3}
\end{array}
\end{gathered}
$$

Where:

$$
\begin{gathered}
Y_{1}=0.04693 X_{1}+0.2381 X_{3} \\
Y_{2}=0.01707 X_{2}+0.281 X_{3}
\end{gathered}
$$




$$
\begin{gathered}
\dot{X}_{1}=-0.06667 X_{1}+0.03125 U_{1} \\
\dot{X}_{2}=-0.04762 X_{2}+0.9563 U_{1} \\
\dot{X}_{3}=-0.04762 X_{3}+U_{2}
\end{gathered}
$$

Convert $\dot{X}_{1}$ to S-Domain from Equation (9)

$$
\begin{gathered}
S X_{1}(s)=-0.06667 X_{1}(s)+0.03125 U_{1} \\
(S+0.06667) X_{1}(s)=0.03125 U_{1} \\
X_{1}(s)=\frac{0.03125}{s+0.06667} U_{1}
\end{gathered}
$$

Convert $\dot{X}_{2}$ to S-Domain from Equation (13)

$$
\begin{gathered}
(S+0.04762) X_{2}(s)=0.9563 U_{1} \\
X_{2}(s)=\frac{0.9563}{s+0.04762} U_{1}
\end{gathered}
$$

Convert $\dot{X}_{3}$ to S-Domain from Equation (14)

$$
\begin{gathered}
S X_{3}(s)=-0.04762 X_{3}(s)+U_{2} \\
(S+0.04762) X_{3}(s)=U_{2} \\
X_{3}(s)=\frac{1}{s+0.04762} U_{2}
\end{gathered}
$$

Equations (12), (13) and (14) used to design state space block diagram of CSTR in MIMO system as shown in figure (1).

$$
S X_{2}(s)=-0.04762 X_{2}(s)+0.9563 U_{1}
$$

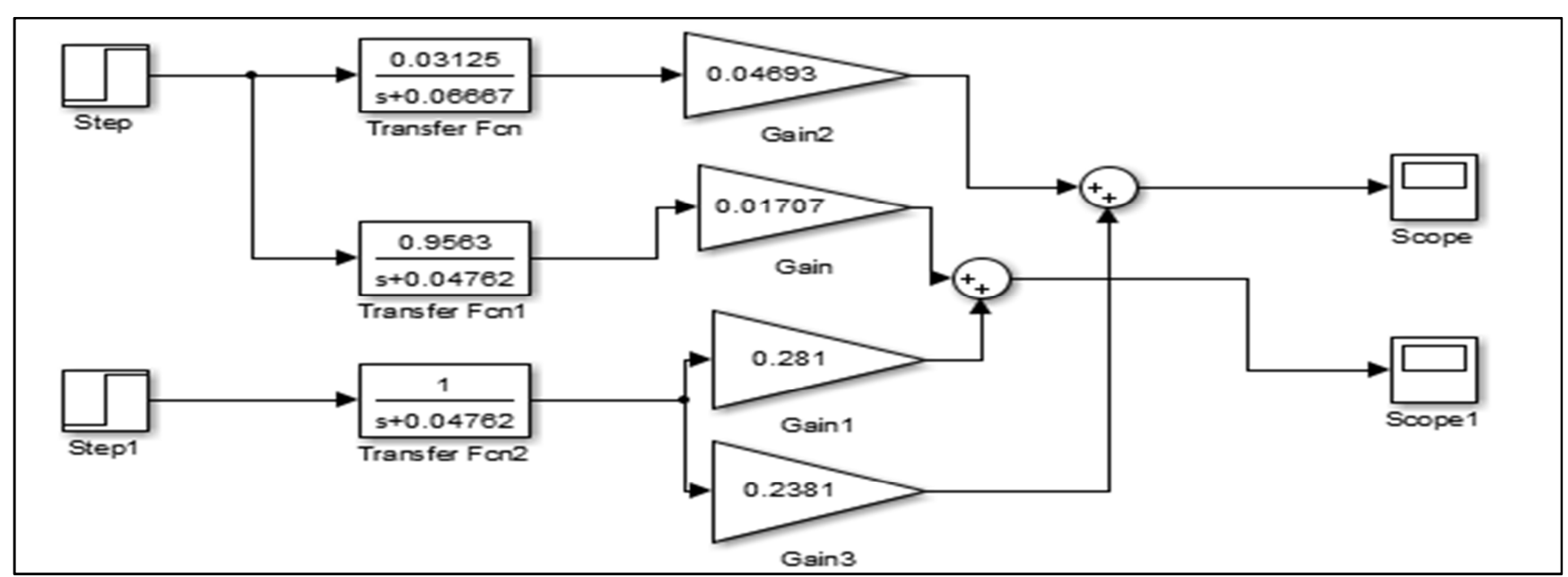

Figure 1. Block diagram of state space modeling.

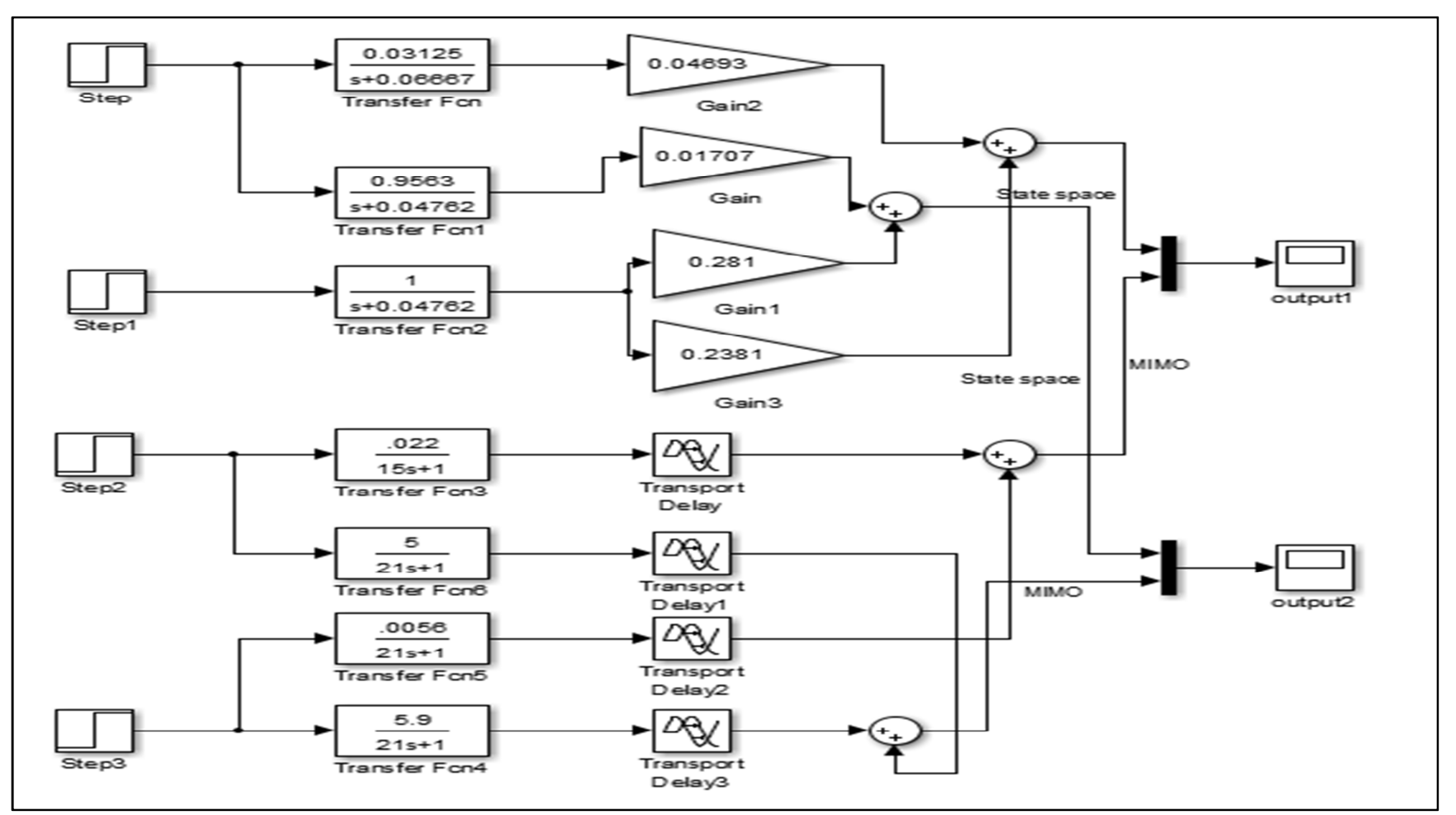

Figure 2. Block diagrams of state space model connected with experimental results. 


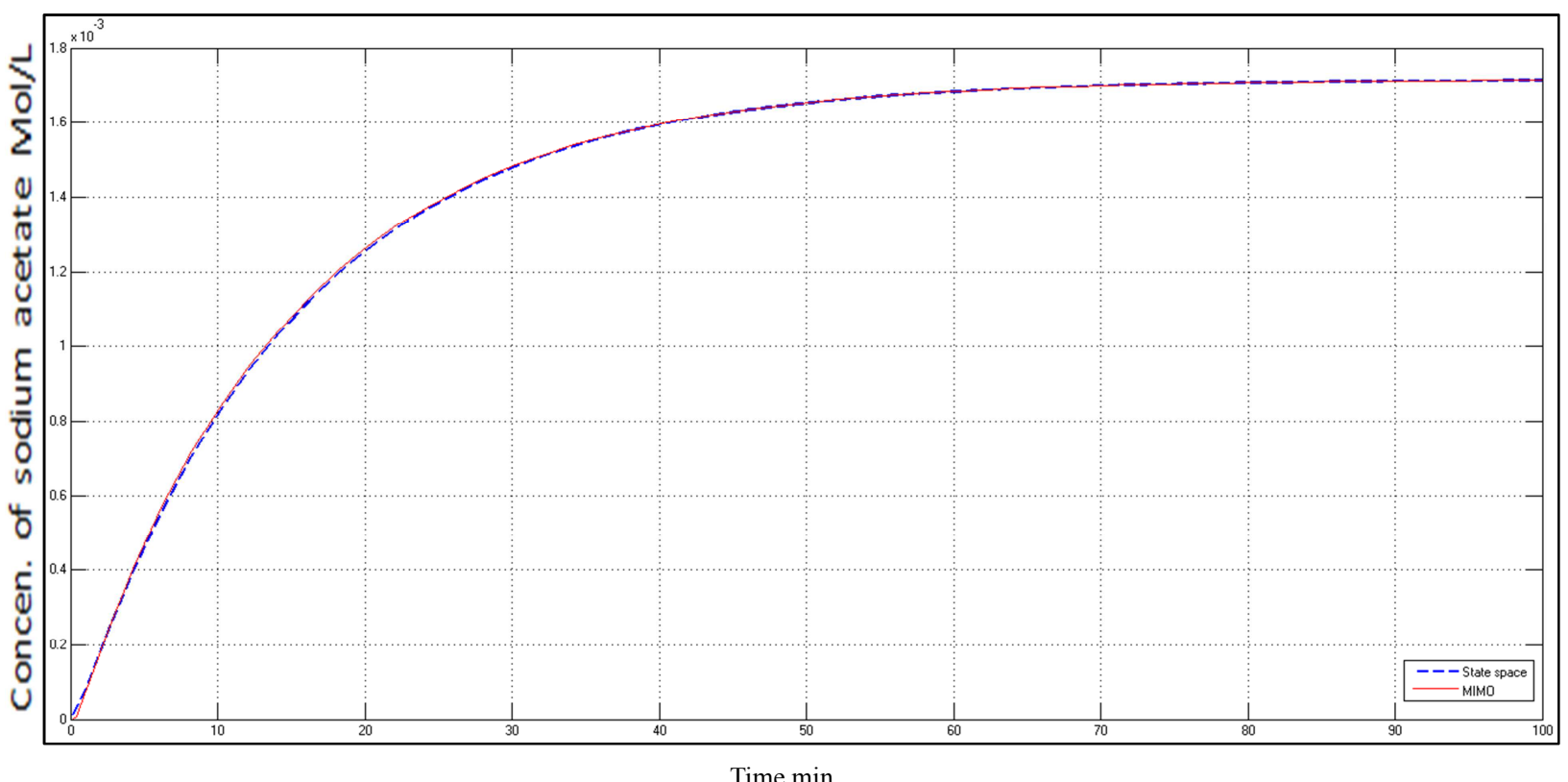

Figure 3. Shows a comparison between the response of the experimental results using $50 \%$ step change in feed of ethyl acetate and the state space model.

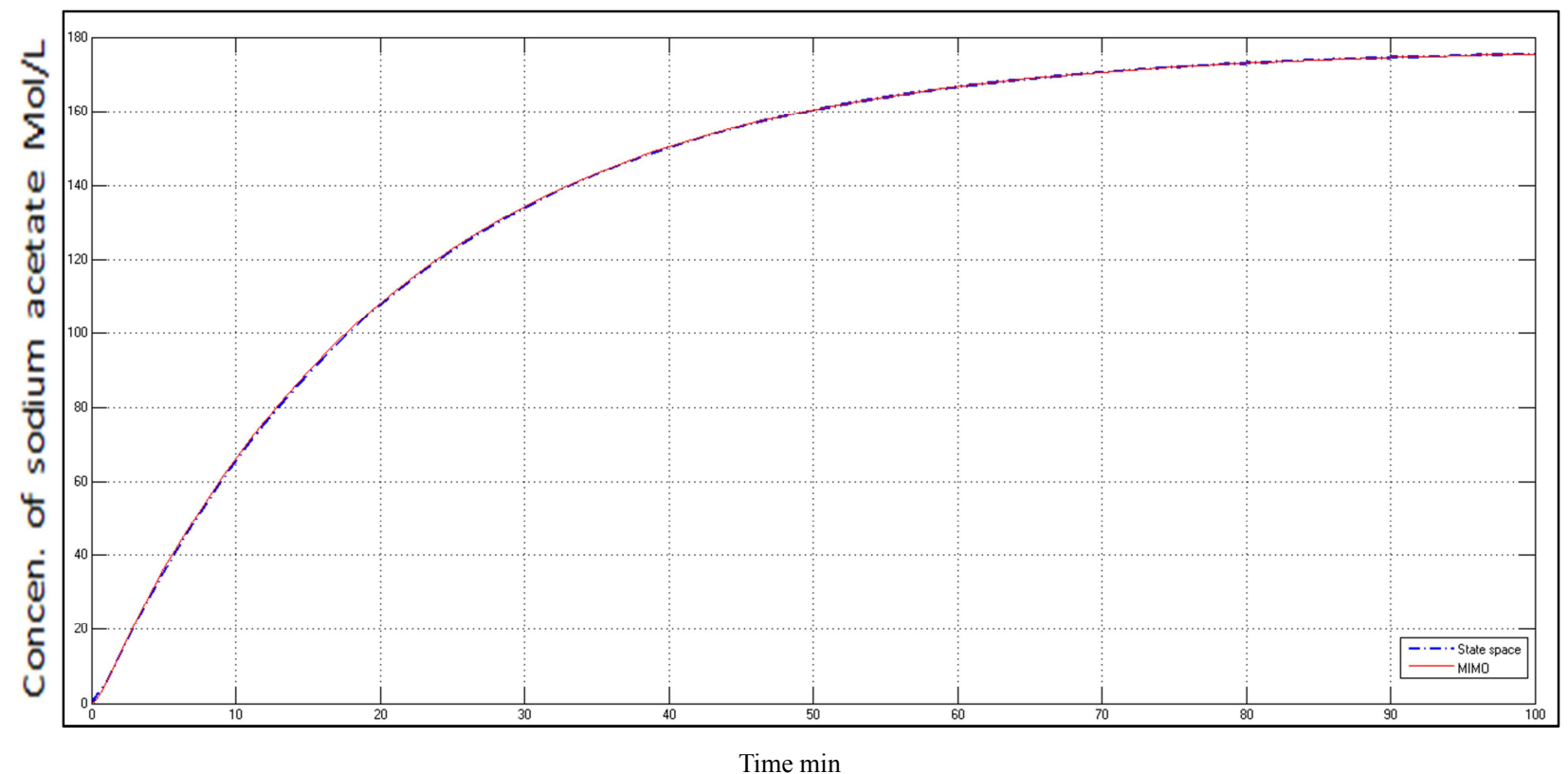

Figure 4. Shows a comparison between the response of the experimental results using 50\% step change in water flow rate in coil and the state space model.

Figure 2 shows the block diagram of state space model connected with experimental results to compare the responses. Figure 3 shows a comparison between the response of the experimental results using 50\% step change in feed of ethyl acetate and the state space model. Figure 4 shows a comparsion between the response of the experimental results using $50 \%$ step change in water flow rate in coil and the state space model.

It's clear that from figures 3 and 4 that great identical was found when using a state space model. Unsatisfied behavior was conducted in previous work in which is a comparison between linearized model and experimental results was shown [13].

\section{Conclusion}

A great improvement was found in using a state space model to analysis the dynamic of the saponifecation process compared with the linearized model on which a awkward results was shown.

\section{References}

[1] http://encyclopedia.che.engin.umich.edu/Pages/Reactors/CST R/CSTR.html. 
[2] P.N.Paraskevopoulos, Modern Control Engineering, Control Engineering Series, 2002, P.78.

[3] L. Robert Williams and A. Douglas Lawrence, Linear StateSpace Control Systems, Ohio University, 2007.

[4] L.M. Patnaik, N. Viswanadham, and I.G. Sarma, State Space Formulation of Ammonia Reactor Dynamics, Computers \& Chemical Engineering, Volume 4, Issue 4, 1980, P. 215-222.

[5] Neil Shephard, Partial Non-Gaussian State Space, Nuffield College, Oxford OX1 1NF, U.K. Biometrika, 1994, P. 115-31.

[6] F. Jeremy Burri, D. Stevan Wilson, and Vasilios I. Manousiouthakis, Infinite Dimensional State-Space Approach to Reactor Network Synthesis: Application to Attainable Region Construction, Computers \& Chemical Engineering, Volume 26, Issue 6, 2002, P. 849-862.

[7] Tapani Raiko and Matti, Learning Nonlinear State-Space Models for Control, Tapani, Neural Networks Research Centre, Helsinki University of Technology, Finland, 2005.

[8] C. Or, Jason L. Speyer, and John Kim, State-Space Approximations of the Orr-Sommerfeld System with Boundary Inputs and Outputs, University of California, Los
Angeles, California, Journal Of Guidance, Control, And Dynamics, Vol. 33, No. 3, 2010.

[9] R.N. Methekar, S.C. Patwardhan, R. Rengaswamy, R.D. Gudi, and V. Prasad, Control of Proton Exchange Membrane Fuel Cells Using Data Driven State Space Models, Chemical Engineering Research and Design, Volume 88, Issue 7, 2010, P. 861-874.

[10] J. Siem Koopman, and J.F. Jacques ,Time Series: State Space Methods, International Encyclopedia of the Social \& Behavioral Sciences, Second Edition, 2015, P. 354-361.

[11] E. Julio Normey-Rico, Control of Dead-time Processes, Advanced Textbooks in Control and Signal processing", 2007, P. 118.

[12] R. Donald Coughanowr, Process Systems Analysis and Control, Department of Chemical Engineering, Drexel University, Second Edition, 1991.

[13] M. Khalid Mousa, Zaynab Esam Dawood, Study on Dynamics of Continuous Stirred Tank Reactor using Multi Input- Multi Output System, International Journal of Engineering Sciences \& Research Technology, 2015. 